DCP-08-04

\title{
Implications of the discovery of a Higgs triplet on electroweak right-handed neutrinos
}

\author{
Alfredo Aranda, ${ }^{1,2 *}$, J. Hernández-Sánchez, ${ }^{2,3 \dagger}$ and P.Q. Hung ${ }^{4 \ddagger}$ \\ ${ }^{1}$ Facultad de Ciencias, CUICBAS, Universidad de Colima, \\ Bernal Díaz del Castillo 340, Colima, Colima, México \\ ${ }^{2}$ Dual C-P Institute of High Energy Physics \\ ${ }^{3}$ Fac. de Cs. de la Electrónica, BUAP, \\ Av. San Claudio y 18 Sur, C. P. 72570, Puebla, Pue., México \\ ${ }^{4}$ Dept. of Physics, University of Virginia, \\ 382 McCormick Road, P.O. box 400714, \\ Charlottesville, Virginia 22904 - 4714, USA.
}

(Dated: October 30, 2018)

\begin{abstract}
Electroweak scale active right-handed neutrinos such as those proposed in a recent model necessitate the enlargement of the SM Higgs sector to include Higgs triplets with doubly charged scalars. The search for and constraints on such Higgs sector has implications not only on the nature of the electroweak symmetry breaking but also on the possibility of testing the seesaw mechanism at colliders such as the LHC and the ILC.
\end{abstract}

\section{INTRODUCTION}

What is the nature of the spontaneous symmetry breaking (SSB) of the Standard Model $(\mathrm{SM})$ ? Assuming that SSB is due to the vacuum expectation value (VEV) of some Higgs field, it goes without saying that one of the most - if not the most - important question that the Large Hadron Collider (LHC) could help us answer regards to the nature of the Higgs mechanism: Is it just one Higgs doublet as in the minimal SM? or is it a complex system involving more than one Higgs doublet and perhaps even Higgs triplets? If one Higgs doublet is sufficient to provide the right kind of SSB for the minimal SM, why would one

\footnotetext{
* Electronic address:fefo@ucol.mx

$\dagger$ Electronic address:jaimeh@ece.buap.mx

$\ddagger$ Electronic address:pqh@virginia.edu
} 
need to invoke a more complicated structure? How well motivated would or should it be? The possible presence of a Higgs content that includes triplets promises to yield a rich "zoo" of electroweak (EW) scalars to be probed at the LHC and ILC.

Although the possibility of having Higgs triplets that obey the quintessential electroweak requirement $\rho=1$ has been studied in detail by [1, 2], the question that always remained is: Why does one need it? In the absence of a direct sign on the nature of the Higgs mechanism, it is certainly fair to contemplate general scenarios as long as they satisfy the electroweak precision data constraints. However, it would be more appealing if there were additional motivations for the use of richer structures such as Higgs triplets. Recently, a model has been proposed [3] in which the right-handed neutrinos that participate in the seesaw mechanism are active in the sense that they are electroweak nonsinglets. As such, if they are not too heavy, they can be produced at colliders with electroweak production cross sections and characteristic signals such as like-sign dileptons. The seesaw mechanism can be tested directly at colliders! In fact, the right-handed neutrinos of [3] are members of SM doublets of mirror leptons and their Majorana masses are intrinsically linked to the electroweak scale through a coupling with a Higgs triplet that develops an electroweak scale VEV. In this model, the sources of the SM SSB are not only Higgs doublet(s) but include Higgs triplets as well: the nature of the SM SSB is intimately linked to the nature of neutrino masses and the possible experimental discovery of the seesaw mechanism at colliders. One cannot fail but to notice the interesting complementarity of a discovery of electroweak scale $\nu_{R}$ 's and that of a Higgs triplet.

In this paper we explore the phenomenology of the model in [3]. The full description of the scalar sector involving the triplet fields can be found in [1, 4, 5, 6], here we briefly review the extension of the basic model to include electroweak neutrinos.

In addition to the SM particle content the model of [3] contains the additional fields shown in table I. There is also an additional global $\mathrm{U}(1)_{M}$ symmetry under which

$$
L_{R}^{M}, e_{L}^{M} \rightarrow e^{i \theta_{M}} L_{R}^{M}, e_{L}^{M} ; \quad \tilde{\chi} \rightarrow e^{-2 i \theta_{M}} \tilde{\chi}, \quad \phi_{S} \rightarrow e^{-i \theta_{M}} \phi_{S},
$$

and all other fields are singlets. This global symmetry was invoked in order to avoid certain terms as indicated below and was explained in detail in [3]. It turns out, however, that when this model is embedded into a Pati-Salam-like quark-lepton unification [7], this global symmetry is no longer needed since the absence of the aforementioned terms is guaranteed 


\begin{tabular}{|c|c|c|}
\hline Additional fields & $\mathrm{SU}(2)_{W}$ & $\mathrm{U}(1)_{Y}$ \\
\hline \hline$L_{R}^{M}=\left(\begin{array}{lll}\nu_{R} & e_{R}^{M}\end{array}\right)$ & $\mathbf{2}$ & -1 \\
\hline$\tilde{\chi}=\left(\begin{array}{lll}\chi^{0} & \chi^{+} & \chi^{++}\end{array}\right)^{T}$ & $\mathbf{3}$ & 2 \\
\hline$\xi=\left(\begin{array}{lll}\xi^{+} & \xi^{0} & \xi^{+}\end{array}\right)^{T}$ & $\mathbf{3}$ & 0 \\
\hline$e_{L}^{M}$ & $\mathbf{1}$ & -2 \\
\hline$\phi_{S}$ & $\mathbf{1}$ & 0 \\
\hline
\end{tabular}

TABLE I: Additional field content of the model with their transformation properties under $\mathrm{SU}(2)_{W}$ and $\mathrm{U}(1)_{Y}$.

by the gauge symmetry of the extended model.

We now briefly comment on the virtues of these assignments: Note that since $\nu_{R}$ is not an $\mathrm{SU}(2)_{L}$ singlet, it does not couple to $\bar{L}_{L} \tilde{\Phi}$. Instead, the Dirac neutrino mass comes from the term

$$
\mathcal{L}_{S}=-g_{s l} \bar{L}_{L} \phi_{S} L_{R}^{M}+\text { h.c. }
$$

which leads to $M_{\nu}^{D}=g_{s l} v_{S}$, where $\left\langle\phi_{S}\right\rangle=v_{S}$ and thus the neutrino Dirac mass is independent of the EW scale [3]. Notice that $\phi_{S}$ is a singlet Higgs field.

Active right-handed neutrinos must have a mass $>M_{Z} / 2$ in order not to contribute to the $Z$ width. This is accomplished with the $Y=2$ triplet $\tilde{\chi}$ through the term

$$
g_{M} L_{R}^{M, T} \sigma_{2} \tau_{2} \tilde{\chi} L_{R}^{M}
$$

which leads to

$$
M_{R}=g_{M} v_{M}
$$

with $\left\langle\chi^{0}\right\rangle=v_{M}$ and where $v_{M}=O\left(\Lambda_{E W}\right)$. This allows to have EW-scale masses for the righthanded neutrinos without having to fine-tune the Yukawa coupling $g_{M}$ to be abnormally small [3].

An important observation is that the $\mathrm{U}(1)_{M}$ symmetry was introduced [3] in order to forbid the terms $g_{L} L_{L}^{T} \sigma_{2} \tau_{2} \tilde{\chi} L_{L}$ and $L_{L}^{T} \sigma_{2} \tau_{2} \tilde{\chi} L_{R}^{M}$ at tree level. A similar result is obtained in an extension of that model [7] where the global $\mathrm{U}(1)_{M}$ is not needed for that purpose. The main consequence of this is that the Dirac mass for the neutrinos comes from $v_{S}$ exclusively 
and the Majorana mass, $M_{L}$, for the left-handed neutrinos arises at the one-loop level and can be much smaller than $M_{R}$.

Taking all of this into consideration one obtains the following Majorana mass matrix:

$$
\mathcal{M}=\left(\begin{array}{ll}
M_{L} & m_{\nu}^{D} \\
m_{\nu}^{D} & M_{R}
\end{array}\right),
$$

where, as we have just mentioned above, $M_{L} \sim \epsilon\left(m_{\nu}^{D}\right)^{2} / M_{R}<10^{-2}\left(m_{\nu}^{D}\right)^{2} / M_{R}$.

We are interested in the scenario where $g_{s l} \sim \mathrm{O}\left(g_{M}\right)$ and $v_{M}>>v_{S}$. In this case, the eigenvalues of $\mathcal{M}$ become $-\left(g_{s l}^{2} / g_{M}\right)\left(v_{S} / v_{M}\right) v_{S}(1-\epsilon)$ and $M_{R}$, where $\epsilon<10^{-2}$. Now, since $v_{M} \sim \Lambda_{E W}$, and using the bound $m_{\nu} \leq 1 \mathrm{eV}$, we have [3]

$$
v_{S} \approx \sqrt{(1 \mathrm{eV}) \times v_{M}} \sim \mathrm{O}\left(10^{5-6} \mathrm{eV}\right) .
$$

So far the $Y=0$ triplet has not played a role since it does not couple to fermions. However, it has been introduced in order to ensure $\rho=1$ at tree level [1]. Note that in principle the parameter $g_{s l}$ is constrained by the neutrino mass spectrum. We work under the premise that the smallness of the Dirac mass is a result of $v_{S}$ and not from a very small coupling. Thus, if $g_{s l} \sim \mathrm{O}(1)$, then $v_{S} \sim 10^{5} \mathrm{eV}$. This amounts to a hierarchy among the scales $v_{S} / \Lambda_{E W} \sim 10^{-6}$ which is however not as severe as the usual hierarchies in GUTS. This has been discussed in full detail in [3, 8].

\section{SCALAR SECTOR}

The kinetic part of the Higgs Lagrangian is

$$
\mathcal{L}_{k i n}=\frac{1}{2} \operatorname{Tr}\left[\left(D_{\mu} \Phi\right)^{\dagger}\left(D^{\mu} \Phi\right)\right]+\frac{1}{2} \operatorname{Tr}\left[\left(D_{\mu} \chi\right)^{\dagger}\left(D^{\mu} \chi\right)\right]+\left|\partial_{\mu} \phi_{S}\right|^{2},
$$

where

$$
\begin{gathered}
\chi=\left(\begin{array}{ccc}
\chi^{0} & \xi^{+} & \chi^{++} \\
\chi^{-} & \xi^{0} & \chi^{+} \\
\chi^{--} & \xi^{-} & \chi^{0 *}
\end{array}\right), \\
D_{\mu} \Phi=\partial_{\mu}+i g(\mathbf{W} \cdot \tau / 2) \Phi-i g^{\prime} \Phi B \tau_{3} / 2 \\
D_{\mu} \chi=\partial_{\mu} \chi+i g \mathbf{W} \cdot \mathbf{t} \chi-i g^{\prime} \chi B t_{3} .
\end{gathered}
$$


As mentioned above, we work under the premise that the hierarchy in neutrino masses comes from the VEV of $\phi_{S}$. This amounts to $v_{S} \sim 10^{5} \mathrm{eV}$ and in turn to a negligible mixing between $\phi_{S}$ and the other scalars. In what follows we neglect such mixing.

The potential (for $\Phi$ and $\chi$ ) to be considered is [1]

$$
\begin{aligned}
V(\Phi, \chi) & =\lambda_{1}\left(\operatorname{Tr} \Phi^{\dagger} \Phi-v_{2}^{2}\right)^{2}+\lambda_{2}\left(\operatorname{Tr} \chi^{\dagger} \chi-3 v_{M}^{2}\right)^{2} \\
& +\lambda_{3}\left(\operatorname{Tr} \Phi^{\dagger} \Phi-v_{2}^{2}+\operatorname{Tr} \chi^{\dagger} \chi-3 v_{M}^{2}\right)^{2} \\
& +\lambda_{4}\left(\operatorname{Tr} \Phi^{\dagger} \Phi \operatorname{Tr} \chi^{\dagger} \chi-2 \operatorname{Tr} \Phi^{\dagger} T^{i} \Phi T^{j} \cdot \operatorname{Tr} \chi^{\dagger} T^{i} \chi T^{j}\right) \\
& +\lambda_{5}\left[3 \operatorname{Tr} \chi^{\dagger} \chi \chi^{\dagger} \chi-\left(\operatorname{Tr} \chi^{\dagger} \chi\right)^{2}\right] .
\end{aligned}
$$

Note that this potential is invariant under $\chi \rightarrow-\chi$. In order for the potential to be positive semidefinite the following conditions must be imposed: $\lambda_{1}+\lambda_{2}+2 \lambda_{3}>0, \lambda_{1} \lambda_{2}+$ $\lambda_{1} \lambda_{3}+\lambda_{2} \lambda_{3}>0, \lambda_{4}>0, \lambda_{5}>0$. Furthermore the potential is invariant under the global symmetry $\mathrm{SU}(2)_{L} \times \mathrm{SU}(2)_{R}$.

When $\chi$ gets a $\operatorname{VEV}\langle\chi\rangle=\operatorname{diag}\left(v_{M}, v_{M}, v_{M}\right)$ it breaks the global symmetry $\mathrm{SU}(2)_{L} \times$ $\mathrm{SU}(2)_{R}$ down to the custodial $\mathrm{SU}(2)_{C}$. It was shown in [1, 2] that the structure of the VEV is dictated by the proper vacuum alignment. Now, using $\langle\Phi\rangle=v_{2} / \sqrt{2}$, the $W$ and $Z$ masses can be obtained from Eq. (7) and are given by $M_{W}=g v / 2$ and $M_{Z}=M_{W} / \cos \theta_{W}$, with

$$
v^{2}=v_{2}^{2}+8 v_{M}^{2},
$$

with $v \approx 246 \mathrm{GeV}$. This gives rise to $\rho=1$ at tree level.

A convenient parametrization can be made by defining $\cos \theta_{H}=c_{H} \equiv v_{2} / v$ and thus $\sin \theta_{H}=s_{H} \equiv 2 \sqrt{2} v_{M} / v$. Using these parameters we can see that $\tan \theta_{H}=t_{H}$ characterizes the amount of the $W$ mass coming from either the doublet or the triplet scalars.

One of the important questions that arises in the model of $[3]$ is the relative magnitude of $v_{M}$ compared with the electroweak scale $v \sim 246 \mathrm{GeV}$. The reason we are interested in this VEV is because the right-handed neutrino Majorana mass is $M_{R}=g_{M} v_{M}$ as shown in Eq. (4) and its search through characteristic signals such as like-sign dilepton events depends crucially on the knowledge of $M_{R}$. As we will see below, the constraints coming from the scalar sector limit the range of allowed values of $\sin \theta_{H}=s_{H} \equiv 2 \sqrt{2} v_{M} / v$, and, consequently, $v_{M}$. One cannot fail but to see the deep relationship between the search for the extended Higgs sector and that for the electroweak-scale active right-handed neutrinos. 
We will use the subsidiary fields:

$$
\begin{aligned}
\phi^{0} & \equiv \frac{1}{\sqrt{2}}\left(v_{2}+\phi^{0 r}+i \phi^{0 i}\right), \quad \chi^{0} \equiv v_{M}+\frac{1}{\sqrt{2}}\left(\chi^{0 r}+i \chi^{0 i}\right), \\
\psi^{ \pm} & \equiv \frac{1}{\sqrt{2}}\left(\chi^{ \pm}+\xi^{ \pm}\right), \quad \zeta^{ \pm} \equiv \frac{1}{\sqrt{2}}\left(\chi^{ \pm}-\xi^{ \pm}\right)
\end{aligned}
$$

for the complex neutral and charged fields, respectively.

The Goldstone bosons are given by

$$
G_{3}^{ \pm}=c_{H} \phi^{ \pm}+s_{H} \psi^{ \pm}, \quad G_{3}^{0}=i\left(-c_{H} \phi^{0 i}+s_{H} \chi^{0 i}\right)
$$

If the potential preserves the $\mathrm{SU}(2)_{C}$ then the fields get arranged in the following manner (based on their transformation properties under the custodial SU(2)):

$$
\begin{aligned}
& \text { five }- \text { plet } \rightarrow H_{5}^{ \pm \pm}, H_{5}^{ \pm}, H_{5}^{0} \leftrightarrow \text { degenerate } \\
& \text { three }- \text { plet } \rightarrow H_{3}^{ \pm}, H_{3}^{0} \leftrightarrow \text { degenerate } \\
& 2 \text { - singlets } \rightarrow H_{1}^{0}, H_{1}^{0 \prime} \leftrightarrow \text { Only these can mix },
\end{aligned}
$$

where

$$
\begin{aligned}
H_{5}^{++} & =\chi^{++}, \quad H_{5}^{+}=\zeta^{+}, \quad H_{3}^{+}=c_{H} \psi^{+}-s_{H} \phi^{+} \\
H_{5}^{0} & =\frac{1}{\sqrt{6}}\left(2 \xi^{0}-\sqrt{2} \chi^{0 r}\right), \quad H_{3}^{0}=i\left(c_{H} \chi^{0 i}+s_{H} \phi^{0 i}\right) \\
H_{1}^{0} & =\phi^{0 r} \\
H_{1}^{0 \prime} & =\frac{1}{\sqrt{3}}\left(\sqrt{2} \chi^{0 r}+\xi^{0}\right)
\end{aligned}
$$

with $H_{5}^{--}=\left(H_{5}^{++}\right)^{*}, H_{5}^{-}=-\left(H_{5}^{+}\right)^{*}, H_{3}^{-}=-\left(H_{3}^{+}\right)^{*}$, and $H_{3}^{0}=-\left(H_{3}^{0}\right)^{*}$. It is also convenient to express the triplet neutral scalar $\chi^{0}$ in terms of the above states, namely

$$
\chi^{0} \equiv v_{M}+\frac{1}{\sqrt{3}} H_{1}^{0 \prime}-\frac{1}{\sqrt{6}} H_{5}^{0}+\frac{1}{\sqrt{2} c_{H}} H_{3}^{0}
$$

where only physical states have been included. Feynman rules for vector boson couplings can be found in [5].

One last comment regarding the scalar potential. As discussed in [1] the potential contains an explicit breaking of the $\mathrm{U}(1)_{M}$ symmetry. This renders the model free of NG bosons and the $\phi_{S}$ mass is independent of $v_{S}$. Furthermore there is a would-be-Majoron with a mass larger than the $\mathrm{Z}$ boson mass. 


\section{COUPLINGS TO MATTER}

In the search for the Higgs scalars discussed in this work, it is important to know what those scalars couple to. The couplings of this extended Higgs sector can be found in [2]. Here we are interested in those couplings which are specific to the model of mirror fermions

of [3]. As we shall see below, they can give rise to very specific signatures such as leptonnumber violating decays. In this section we obtain the Feynman rules for scalar fermion couplings including the mirror fermions.

In the case of SM fermions, we have the usual Yukawa interactions

$$
\mathcal{L}_{Y}=-h_{i j} \bar{\Psi}_{L i} \Phi \Psi_{R j}+\text { h.c. }
$$

The Feynman rules obtained from this Lagrangian become [5]

$$
\begin{aligned}
g_{H_{1}^{0} q \bar{q}} & =-i \frac{m_{q} g}{2 m_{W} c_{H}}(q=t, b) \\
g_{H_{3}^{0} t \bar{t}} & =i \frac{m_{t} g s_{H}}{2 m_{W} c_{H}} \gamma_{5}, \\
g_{H_{3}^{0} b \bar{b}} & =-i \frac{m_{b} g s_{H}}{2 m_{W} c_{H}} \gamma_{5}, \\
g_{H_{3}^{-} t \bar{b}} & =i \frac{g s_{H}}{2 \sqrt{2} m_{W} c_{H}}\left(m_{t}\left(1+\gamma_{5}\right)-m_{b}\left(1-\gamma_{5}\right)\right),
\end{aligned}
$$

where third generation notation is used for quarks and similar expressions apply to leptons.

For mirror fermions we need to consider the terms

$$
\mathcal{L}_{M 1}=-g_{l}^{M} \bar{L}_{R}^{M} \Phi e_{L}^{M}+\text { h.c. }
$$

and

$$
\mathcal{L}_{M 2}=-g_{M} L_{R}^{M, T} \sigma_{2} \tau_{2} \tilde{\chi} L_{R}^{M} .
$$

This leads to the following Feynman rules: from Eq. (22) one obtains

$$
\begin{aligned}
g_{H_{3}^{+} \nu \bar{l}^{M}} & =i \frac{m_{l}^{M} g s_{H}}{2 \sqrt{2} m_{W} c_{H}}\left(1-\gamma_{5}\right), \\
g_{H_{1}^{0} l^{M} \bar{l}^{M}} & =-i \frac{m_{l}^{M} g}{2 \sqrt{2} m_{W} c_{H}}, \\
g_{H_{3}^{0} l^{M} \bar{l}^{M}} & =i \frac{m_{l}^{M} g s_{H}}{2 \sqrt{2} m_{W} c_{H}} \gamma_{5},
\end{aligned}
$$

where

$$
m_{l}^{M}=g_{l}^{M} \frac{v_{2}}{\sqrt{2}}=\frac{\sqrt{2} m_{w} c_{H} g_{l}^{M}}{g}
$$


and from Eq. (23) we get

$$
\begin{aligned}
g_{H_{1}^{0 \prime} \nu_{R} \nu_{R}} & =i \frac{g_{M} \sigma_{2} \otimes\left(1+\gamma_{5}\right)}{2 \sqrt{3}}, \\
g_{H_{5}^{0} \nu_{R} \nu_{R}} & =-i \frac{g_{M} \sigma_{2} \otimes\left(1+\gamma_{5}\right)}{\sqrt{6}} \\
g_{H_{3}^{0} \nu_{R} \nu_{R}} & =i \frac{g_{M} \sigma_{2} \otimes\left(1+\gamma_{5}\right)}{\sqrt{2} c_{H}}, \\
g_{H_{5}^{+} \nu_{R} e^{M,+}} & =i \frac{g_{M} \sigma_{2} \otimes\left(1+\gamma_{5}\right)}{\sqrt{2}}, \\
g_{H_{3}^{+} \nu_{R} e^{M,+}} & =i \frac{g_{M} \sigma_{2} \otimes\left(1+\gamma_{5}\right)}{\sqrt{2} c_{H}} .
\end{aligned}
$$

There are also couplings of SM leptons with their mirrors through the term in Eq. (2), i.e.

$$
\begin{aligned}
& g_{\nu_{l} \bar{\nu}_{l} \phi_{S}^{r}}=-i \frac{g_{s l}}{\sqrt{2}}, \quad g_{\nu_{l} \bar{\nu}_{l} \phi_{S}^{i}}=\frac{g_{s l}}{\sqrt{2}} \gamma_{5}, \\
& g_{l \bar{l}^{M} \phi_{S}^{r}}=-i \frac{g_{s l}}{2 \sqrt{2}}\left(1-\gamma_{5}\right), \quad g_{l \bar{l}^{M} \phi_{S}^{i}}=\frac{g_{s l}}{2 \sqrt{2}}\left(1-\gamma_{5}\right),
\end{aligned}
$$

where we have used the definition $\phi_{S}=v_{S}+\frac{1}{\sqrt{2}}\left(\phi_{S}^{r}+i \phi_{S}^{i}\right)$.

A detailed and complete study of the lepton sector of this model has been presented in [8]. In this paper we concentrate on the scalar sector phenomenology specific to this model.

\section{NUMERICAL ANALYSIS}

\section{A. Scalar sector}

In this section we explore the parameter space of the model. We begin by studying the scalar mass spectrum.

The first observation is that the value of $\sin \theta_{H}$ has an upper bound coming from from the

constraint [9] $\tan \theta_{H} \leq 2$. It also has a lower bound coming from the right-handed neutrino mass scale, i.e. $M_{R}>m_{Z} / 2$. Since $M_{R}=g_{M} v_{M}$, this translates into $g_{M} v_{M}<45.6 \mathrm{GeV}$. The lower bound on $\sin \theta_{H}$ comes from finding the lowest allowable value for $v_{M}$. If one uses the simple-minded perturbative requirement $g_{M}^{2} / 4 \pi<1$, one obtains $v_{M}>12.9 \mathrm{GeV}$. Thus we restrict our study to the range

$$
0.15 \leq \sin \theta_{H} \leq 0.89
$$


Equivalently, Eq. (28) can be expressed in terms of the bounds on $v_{M}$ and $v_{2}$ namely

$$
\begin{gathered}
12.9 \mathrm{GeV}<v_{M}<77.4 \mathrm{GeV}, \\
243.3 \mathrm{GeV}>v_{2}>112.2 \mathrm{GeV} .
\end{gathered}
$$

As we have mentioned above, the restrictions on $\sin \theta_{H}$ and consequently on $v_{M}$, have interesting consequences on the mass range of the electroweak-scale active right-handed neutrinos.

We now consider the parameters in Eq. (11) and explore two general possibilities: Either there is no hierarchy among the parameters and treat them on equal footing, or we assume that all parameters involving triplet fields (including those which mix triplet and doublet fields) are suppressed with respect to those that involve only doublet fields. Furthermore whenever a parameter is not suppressed it is assumed to be of order one and by this we mean that the parameter is arbitrarily chosen to be in the range $(0.5-2)$.

Bounds from unitarity [10] are incorporated through the following relations:

$$
\begin{aligned}
m_{H 3} & \leq 400 \mathrm{GeV}, \\
m_{\chi} & \leq \sqrt{3} m_{H 3}, \\
m_{\text {light }} & \leq 270 \mathrm{GeV},
\end{aligned}
$$

where $m_{\text {light }}$ stands for the lightest scalar state. There is also a bound in the $m_{H_{1}^{0}}-m_{H_{1}^{0^{\prime}}}$ plane due to unitarity. It amounts to require the heavier of the two to be less than $(700-550) \mathrm{GeV}$ when the lighter is in the range of $(0-300) \mathrm{GeV}$.

Lastly we incorporate the $115 \mathrm{GeV}$ LEP lower bound on the lightest scalar mass, however we also contemplate the possibility described in [11] that the lightest Higgs might have escaped detection and could be very light indeed.

We proceed by analyzing some specific cases. Figure 1 shows the situation when there is no hierarchy among the parameters in the scalar potential. They are all of $\mathrm{O}(1)$ and taken to be in the lower part of the arbitrarily chosen $\mathrm{O}(1)$ range. It can be seen from the figure that in this case the allowed range for $\sin \theta_{H}$ is $0.3<\sin \theta_{H}<0.65$ where the lower number refers to the LEP bound while the larger number refers to the unitarity constraint on the mass of the lightest neutral scalar.

Figure 2 shows a similar case with no hierarchy but with all parameters in the upper part of the $\mathrm{O}(1)$ range. Here the allowed range is shifted downward compared with the previous bounds, namely $0.17<\sin \theta_{H}<0.35$. 
The same situation occurs for the case of intermediate values with no hierarchy as can be seen in figure 3 where now one has $0.22<\sin \theta_{H}<0.48$. Thus, if all parameters in the potential are taken of the same order, i.e. no hierarchy, then the allowed range for $\sin \theta_{H}$ decreases as those parameters go from $\sim 0.5$ to $\sim 2$.

There is an interesting case where we allow for a small hierarchy among some of the parameters, namely if we let $\lambda_{4}$ be larger than the other parameters, while still all of them in the $\mathrm{O}(1)$ range, then the situation is that of figure 4 .

Figures 5 and 6 show the cases where there is a hierarchy among $\lambda_{1}$ and the other parameters. Here $\lambda_{1}$, which is related to the doublet fields exclusively, is taken to be of $\mathrm{O}(1)$ while the rest are suppressed by a factor of 10. Again, this factor is arbitrary. Figure 5 presents the situation where $\lambda_{1}$ lies in the lower side of the $\mathrm{O}(1)$ range and it can be seen that the spectrum satisfies all bounds for $\sin \theta_{H}>0.6$, except for the LEP bound. Figure 6 shows the result for $\lambda_{1}$ in the upper part of the $\mathrm{O}(1)$ range and in this case the spectrum satisfies the bounds (except for LEP) for all the $\sin \theta_{H}$ range. It is interesting that these scenarios could fall into the category described in [11] where there is a light scalar unobserved by LEP. One way to study this possibility is to consider Higgs production in $e^{+} e^{-}$collisions, i.e. through the Higgs-strahlung processes $e^{+} e^{-} \rightarrow H_{i}^{0} Z^{0}$, whose cross sections can be expressed in terms of the SM Higgs boson (herein denoted by $\phi_{S M}^{0}$ ) production formula and the Higgs- $Z^{0} Z^{0}$ coupling as follows [11]:

$$
\sigma_{H_{i}^{0} Z}=R_{H_{i}^{0} Z^{0} Z^{0}}^{2} \sigma_{H_{i}^{0} Z}^{S M}
$$

with

$$
R_{H_{i}^{0} Z^{0} Z^{0}}^{2}=\frac{g_{H_{i}^{0} Z^{0} Z^{0}}^{2}}{g_{\phi_{S M}^{0} Z^{0} Z^{0}}^{2}}
$$

where $g_{H_{i}^{0} Z^{0} Z^{0}}^{2}$ is the $H_{i}^{0} Z^{0} Z^{0}$ coupling in our model and $g_{\phi_{S M}^{0} Z^{0} Z^{0}}^{2}$ is the $\phi_{S M}^{0} Z^{0} Z^{0}$ SMcoupling with the relation

$$
\sum_{i=1}^{3} g_{H_{i}^{0} Z^{0} Z^{0}}^{2}=g_{\phi_{S M}^{0} Z^{0} Z^{0}}^{2} .
$$

In particular, for the lightest scalar in the present model, $R_{h^{0} Z^{0} Z^{0}}^{2}$ is given by:

$$
R_{h^{0} Z^{0} Z^{0}}^{2}=\left(-c_{H} s_{\alpha}+\frac{2 \sqrt{2}}{\sqrt{3}} s_{H} c_{\alpha}\right)^{2}
$$


where $\alpha$ is the mixing angle that relates the physical states $h^{0}, H^{0}$ to $H_{1}^{0}, H_{1}^{\prime 0}$ :

$$
\begin{aligned}
H_{1}^{0} & =c_{\alpha} H^{0}-s_{\alpha} h^{0}, \\
H_{1}^{\prime 0} & =s_{\alpha} H^{0}+c_{\alpha} h^{0}, \\
\tan 2 \alpha & =\frac{2 m_{12}^{2}}{m_{11}^{2}-m_{12}^{2}},
\end{aligned}
$$

where $m_{i j}$ denote the mass-squared matrix elements of the two scalars $H_{1}^{0}, H_{1}^{0^{\prime}}$ given by:

$$
M_{H_{1}^{0}, H_{1}^{0^{\prime}}}^{2}=\left(\begin{array}{cc}
8 c_{H}^{2}\left(\lambda_{1}+\lambda_{3}\right) & 2 \sqrt{6} s_{H} c_{H} \lambda_{3} \\
2 \sqrt{6} s_{H} c_{H} \lambda_{3} & 3 s_{H}^{2}\left(\lambda_{2}+\lambda_{3}\right)
\end{array}\right) .
$$

It is also useful to express the Majorana coupling of $\nu_{R}$ to the physical states $H^{0}$ and $h^{0}$, namely

$$
\begin{aligned}
g_{H^{0} \nu_{R} \nu_{R}} & =i \frac{g_{M} s_{\alpha} \sigma_{2} \otimes\left(1+\gamma_{5}\right)}{2 \sqrt{3}}, \\
g_{h^{0} \nu_{R} \nu_{R}} & =i \frac{g_{M} c_{\alpha} \sigma_{2} \otimes\left(1+\gamma_{5}\right)}{2 \sqrt{3}} .
\end{aligned}
$$

The bounds on the neutral Higgs bosons masses are then expressed in terms of the LEP2 bounds for $R_{H_{i}^{0} Z^{0} Z^{0}}^{2}$ [1]]. We find that large regions of the parameter space of our model are excluded as can be seen in Table II. By "parameter space of our model" we mean the region in which the mass of the lightest scalar is situated below the LEP bound. We have defined as "marginal regions" those cases that almost pass the LEP2 bounds on the neutral Higgs mass, i.e., when $m_{h^{0}} \sim 110 \mathrm{GeV}$ and/or when $R_{h^{0} Z^{0} Z^{0}}^{2}$ is almost consistent with the experimental bounds (see case $\mathrm{c}$ in Table II). Our motivation for this definition is that once the complete calculation of the one-loop radiative corrections to the mass of the neutral Higgs boson is considered, one could expect an enhancement for its mass, thereby allowing it to satisfy the experimental bounds. It is known that the inclusion of radiative corrections can alter significantly the (lightest) neutral CP-even Higgs mass, for example in supersymmetric models as MSSM [12] and MSSM+Higgs triplets[13].

\section{B. Signals from the Higgs triplet neutral scalars}

Interesting and unusual signatures come from the presence of mirror fermions. In particular, we are interested in signals that show lepton number violation such as like-sign dilepton 


\begin{tabular}{|c|c|c|c|c|}
\hline a) & $0.34<s_{H}<0.87$ & $45 \mathrm{GeV}<m_{h^{0}}<116 \mathrm{GeV}$ & $\begin{array}{l}0.3<R_{h^{0} Z^{0} Z^{0}}^{2} \text { for } m_{h^{0}}<100 \mathrm{GeV} \\
1.5<R_{h^{0} Z^{0} Z^{0}}^{2} \text { for } m_{h^{0}}<116 \mathrm{GeV}\end{array}$ & Excluded by $R_{h^{0} Z^{0} Z^{0}}^{2}$ \\
\hline b) & $0.34<s_{H}<0.89$ & $44 \mathrm{GeV}<m_{h^{0}}<116 \mathrm{GeV}$ & $1.5<R_{h^{0} Z^{0} Z^{0}}^{2}$ & Excluded by $R_{h^{0} Z^{0} Z^{0}}^{2}$ \\
\hline c) & $0.34<s_{H}<0.7$ & $55 \mathrm{GeV}<m_{H_{1}^{ \pm}}<110 \mathrm{GeV}$ & $\begin{array}{l}0.19<R_{h^{0} Z^{0} Z^{0}}^{2} \text { for } m_{h^{0}}<110 \mathrm{GeV} \\
R_{h^{0} Z^{0} Z^{0}}^{2}<0.29 \text { for } m_{h^{0}} \sim 110 \mathrm{GeV}\end{array}$ & $\begin{array}{c}\text { Allowed by } R_{h^{0}}^{2} Z^{0} Z^{0} \\
\text { only when } m_{h^{0}} \sim 110 \mathrm{GeV}\end{array}$ \\
\hline
\end{tabular}

TABLE II: Analysis of $R_{h^{0} Z^{0} Z^{0}}^{2}$ at tree level consistent with LEP. We consider experimental limits allowed by LEP2 for charged and neutral Higgs bosons for the cases a) $\lambda_{1}=1.5, \lambda_{2}=0.05$ and $\left.\lambda_{3}=0.05, \mathrm{~b}\right) \lambda_{1}=0.5, \lambda_{2}=0.05$ and $\left.\lambda_{3}=0.05, \mathrm{c}\right) \lambda_{1}=0.1, \lambda_{2}=0.1$ and $\lambda_{3}=0.1$.

events. The Lagrangian in Eq. (3) shows the coupling of mirror fermions with the $Y=2$ triplet Higgs field. There is no coupling with the SM leptons which is forbidden either by the $\mathrm{U}(1)_{M}$ symmetry of the model [3] or by embedding it in a Pati-Salam type of quark-lepton unification [7]. This coupling which is obviously lepton-number violating should show up in the decays of triplet scalars in an interesting way.

One can have the following decays: $H^{0}, h^{0}, H_{5}^{0}, H_{3}^{0} \rightarrow \nu_{R} \nu_{R}$. The couplings of $\nu_{R}$ to $H^{0}, h^{0}$ are given in Eq. (42) and to $H_{5}^{0}, H_{3}^{0}$ in Eq. (26). Depending on the mass difference between $\nu_{R}$ 's and the charged mirror leptons $e_{R}^{M}$ 's, the subsequent decay of each $\nu_{R}$ is $\nu_{R} \rightarrow e_{R}^{M}+W^{+} \rightarrow e_{L}+\phi_{S}+W^{+}$, where $e_{R}^{M}$ could be real or virtual (as well as W's). Since $\nu_{R}$ is its own antiparticle one eventually has $H_{\text {neutral }} \rightarrow e_{L}^{\mp}+e_{L}^{\mp}+\phi_{S}+\phi_{S}+W^{ \pm}+W^{ \pm}$, where $H_{\text {neutral }}=H^{0}, h^{0}, H_{5}^{0}, H_{3}^{0}$. This is an example of a lepton-number violating like-sign dilepton decay mode of the neutral scalars. The decay width has a form which is identical to Eq. (43) except for a factor of $1 / 2$ due to the Majorana nature of $\nu_{R}$.

\section{Signals from $\chi^{++}$Decays}

The presence of a doubly charged Higgs in this model provides with interesting phenomenology. Furthermore, the phenomenology of this model is specific and different from that of the general two triplets model due to the following observations:

- Due to the $\mathrm{U}(1)_{M}$ symmetry of the model or its embedding in a Pati-Salam type of quark-lepton unification, the term proportional to $l_{l}^{T} \sigma_{2} \tau_{2} \tilde{\chi} l_{L}$ is not allowed and thus the decay $\Gamma\left(\chi^{++} \rightarrow l^{+} l^{+}\right)$is not present.

- The presence of mirror fermions and $\phi_{S}$ allows for the decays $\Gamma\left(\chi^{++} \rightarrow l_{i}^{M} l_{j}^{M}\right)$ and 
$\Gamma\left(\chi^{++} \rightarrow l \phi_{S} l_{M}\right)$ or even $\Gamma\left(\chi^{++} \rightarrow l l \phi_{S} \phi_{S}\right)$

We now present the expressions for the relevant $\chi^{++}$decays. If $\chi^{++}$is very heavy, it can have the following decays:

- $\chi^{++} \rightarrow l_{M} l_{M}$

$$
\Gamma\left(\chi^{++} \rightarrow l_{i}^{M} l_{j}^{M}\right)=\frac{g_{M}^{2} m_{\chi}}{16 \pi\left(1+\delta_{i j}\right)}\left(1-4 r_{M}^{2}\right)^{1 / 2}
$$

where $r_{M}=m_{l}^{M} / m_{\chi}$ and then

$$
\Gamma\left(l^{M} \rightarrow l \phi_{S}^{r}\right)=\frac{g_{s l}^{2} m_{l}^{M}}{64 \pi}\left(1-\frac{m_{S}^{2}}{\left(m_{l}^{M}\right)^{2}}\right)\left|1-\frac{m_{S}^{2}}{\left(m_{l}^{M}\right)^{2}}\right| .
$$

- $\chi^{++} \rightarrow W^{+} W^{+}$

$$
\Gamma\left(\chi^{++} \rightarrow W^{+} W^{+}\right)=\frac{g^{4} v_{M}^{2}}{32 \pi r_{W}^{4} m_{\chi}}\left(1-4 r_{W}^{2}\right)^{1 / 2}\left(1-4 r_{W}^{2}+12 r_{W}^{4}\right),
$$

where $r_{W} \equiv m_{W} / m_{\chi}$, and $v_{M}=\left\langle\chi^{0}\right\rangle$.

- $\chi^{++} \rightarrow H_{3}^{+} W^{+}$

$$
\Gamma\left(\chi^{++} \rightarrow H_{3}^{+} W^{+}\right)=\frac{c_{H}^{2} g^{2} m_{H_{3}}}{32 \pi x^{3} y^{2}} F_{1}(x, y) F_{2}(x, y),
$$

where $x \equiv m_{\chi} / m_{H_{3}}, y \equiv m_{W} / m_{H_{3}}$, and

$$
\begin{aligned}
& F_{1}(x, y)=1+x^{4}-3 y^{2}+2 y^{4}-2 x^{2}\left(1+y^{2}\right), \\
& F_{2}(x, y)=\left(x^{4}+\left(y^{2}-1\right)^{2}-2 x^{2}\left(1+y^{2}\right)\right)^{1 / 2} .
\end{aligned}
$$

For intermediate $\chi^{++}$masses we can have the following three body decays (most relevant ones):

- $\chi^{++} \rightarrow W^{+*} W^{+} \rightarrow W^{+} l^{+} \nu_{l}$

$$
\Gamma\left(\chi^{++} \rightarrow l^{+} \nu W^{+}\right)=\frac{g^{4} s_{H}^{2} m_{\chi}}{12(8 \pi)^{3} r_{W}^{4}}\left(1-4 r_{W}^{2}+24 r_{W}^{4}\right) .
$$

- $\chi^{++} \rightarrow W^{+*} H_{3}^{+} \rightarrow H_{3}^{+} l^{+} \nu_{l}$

$$
\Gamma\left(\chi^{++} \rightarrow l^{+} \nu H_{3}^{+}\right)=\frac{g^{2} c_{H}^{2} m_{\chi}}{12(8 \pi)^{3} r_{W}^{4}}\left(1-12 r_{H 3}^{2}\right),
$$

with $r_{H 3}=m_{H 3} / m_{\chi}$.

- $\chi^{++} \rightarrow l_{M}^{*} l_{M} \rightarrow l_{M} l^{+} \phi_{S}$

$$
\Gamma\left(\chi^{++} \rightarrow l^{+} \phi_{S} l_{M}\right)=\frac{3 g_{M}^{2} g_{s l}^{2} m_{\chi}}{(16 \pi)^{3} r_{M}^{4}}\left(1+4 r_{M}^{2}-3 r_{S}^{2}\right)
$$

where $r_{S}=m_{S} / m_{\chi}$. 


\section{Branching Ratios}

Using the previous expressions we can compute the branching ratios. In the following analysis we have made the following assumptions:

- $g_{M}$ and $g_{s l}$ are proportional to the identity matrix and so, in each of the expressions above, $g_{M}$ and $g_{s l}$ represent numbers.

- The model requires $g_{s l}^{2} / g_{M} \sim \mathrm{O}(1)$. We have chosen numbers of $\mathrm{O}(1)$ for both couplings and for the numerical results presented below they have been set to $g_{M}=0.7$ and $g_{s l}=0.8$.

Given these assumptions we compute the following branching ratios: $B\left(\chi^{++} \rightarrow l_{M}^{+} l_{M}^{+}\right)$, $B\left(\chi^{++} \rightarrow W^{+} W^{+}\right), B\left(\chi^{++} \rightarrow H_{3}^{+} W^{+}\right), B\left(\chi^{++} \rightarrow l^{+} \nu W^{+}\right)$and $B\left(\chi^{++} \rightarrow l^{+} \phi_{S} l_{M}^{+}\right)$. Note that from Eq. (48) we could compute the corresponding branching ratio, however in order to satisfy the unitarity condition in Eq. (31) this decay cannot take place in the model.

Figure 7 shows the branching ratios for three different values of $\sin \theta_{H}$ and for small values of the mirror fermions masses (taken to be degenerate) $m_{l M}=50 \mathrm{GeV}$. We can see that the dominant one always corresponds to $B\left(\chi^{++} \rightarrow l_{M} l_{M}\right)$, while the relative dominance of the other channels depends on $\sin \theta_{H}$.

Similar results are obtained for larger $m_{l M}$ as can be seen in figure 8 where we show the branching ratios for $m_{l M}=100 \mathrm{GeV}$.

\section{CONCLUSIONS}

We argue that the study of models with extended scalar sectors involving Higgs triplets is well motivated. We study the phenomenology of a model that, using Higgs triplets, can relate both EWSB and neutrino mass generation using an electroweak scale seesaw mechanism. This can in principle make the seesaw mechanism testable at colliders. The model offers a rich scalar phenomenology involving mirror fermions, a single scalar and the usual charged Higgs processes of extended Higgs models, in particular the doubly charged Higgs. We have studied these processes in detail and have computed the branching ratios for the doubly charged Higgs. We find that for all the allowed parameter space, the dominant decay is to 
the mirror fermions. The existence of this decay would provide a clean signature in favor of this scenario.

\section{Acknowledgments}

A.A. acknowledges support from CONACYT and SNI. A.A. and PQH also acknowledge the Aspen Center for Physics for their hospitality while part of this work was being done. J. H-S. was supported by CONACYT(México) under the grant J50027-F and by PROMEPgrant (México). PQH is supported in parts by the US Department of Energy under grant No. DE-A505-89ER40518.

[1] M. S. Chanowitz and M. Golden, Phys. Lett. B 165, 105 (1985).

[2] H. Georgi and M. Machacek, Nucl. Phys. B 262, 463 (1985).

[3] P. Q. Hung, Phys. Lett. B 649, 275 (2007) arXiv:hep-ph/0612004.

[4] E. Accomando et al., arXiv:hep-ph/0608079.

[5] J. F. Gunion, R. Vega and J. Wudka, Phys. Rev. D 42, 1673 (1990).

[6] T. Han, B. Mukhopadhyaya, Z. Si and K. Wang, arXiv:0706.0441 [hep-ph].

[7] P. Q. Hung, Nucl. Phys. B 805, 326 (2008) arXiv:0805.3486[hep-ph].

[8] P. Q. Hung, Phys. Lett. B 659, 585 (2008) arXiv:0711.0733 [hep-ph]].

[9] H. E. Haber and H. E. Logan, Phys. Rev. D 62, 015011 (2000) arXiv:hep-ph/9909335].

[10] M. Aoki and S. Kanemura, Phys. Rev. D 77, 095009 (2008) arXiv:0712.4053 [hep-ph]].

[11] See Table 14 and discussion in S. Schael et al. [ALEPH Collaboration and DELPHI Collaboration and L3 Collaboration and ], Eur. Phys. J. C 47, 547 (2006) arXiv:hep-ex/0602042.

[12] J. R. Ellis, G. Ridolfi and F. Zwirner, Phys. Lett. B 257, 83 (1991) and Phys. Lett. B 262, 477 (1991).

[13] J. L. Diaz-Cruz, J. Hernandez-Sanchez, S. Moretti and A. Rosado, Phys. Rev. D 77, 035007 (2008) arXiv:0710.4169 [hep-ph]]. 


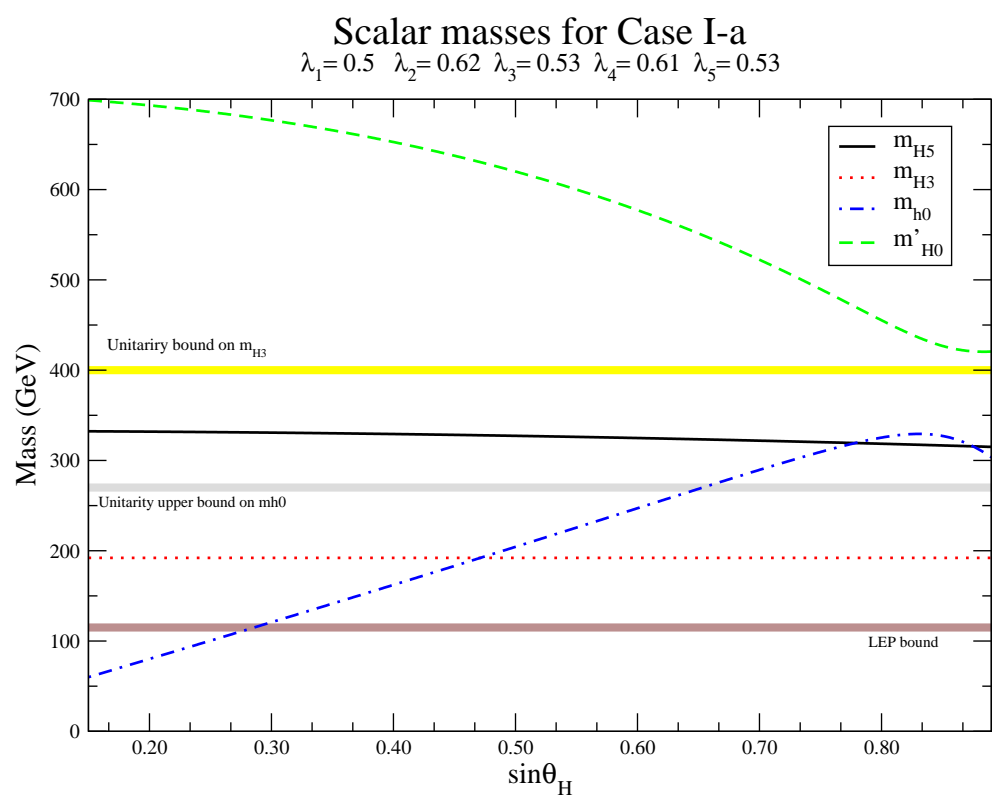

FIG. 1: Scalar mass spectrum for the case where there are no hierarchies among the parameters in the scalar potential. All parameters are in the lower side of the arbitrarily chosen $\mathrm{O}(1)$ range. We explicitly show the upper unitarity bound on the lightest scalar $m_{h^{0}}$ 


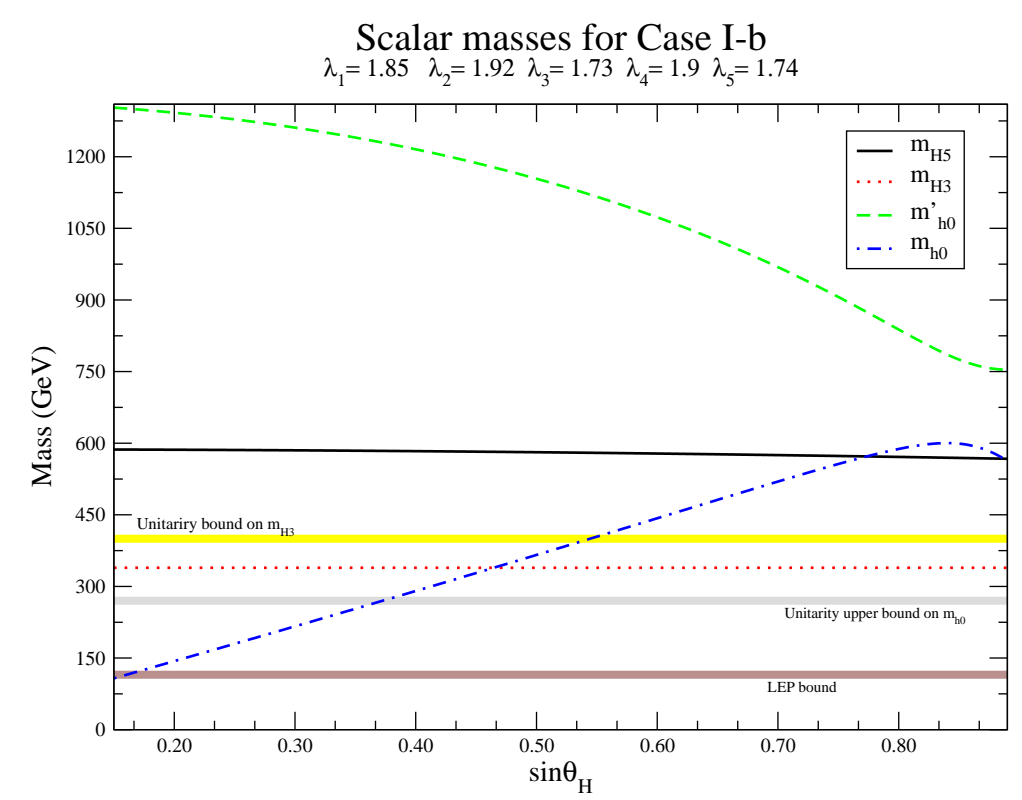

FIG. 2: Scalar mass spectrum for the case where there are no hierarchies among the parameters in the scalar potential. All parameters are in the upper side of the arbitrarily chosen $\mathrm{O}(1)$ range.

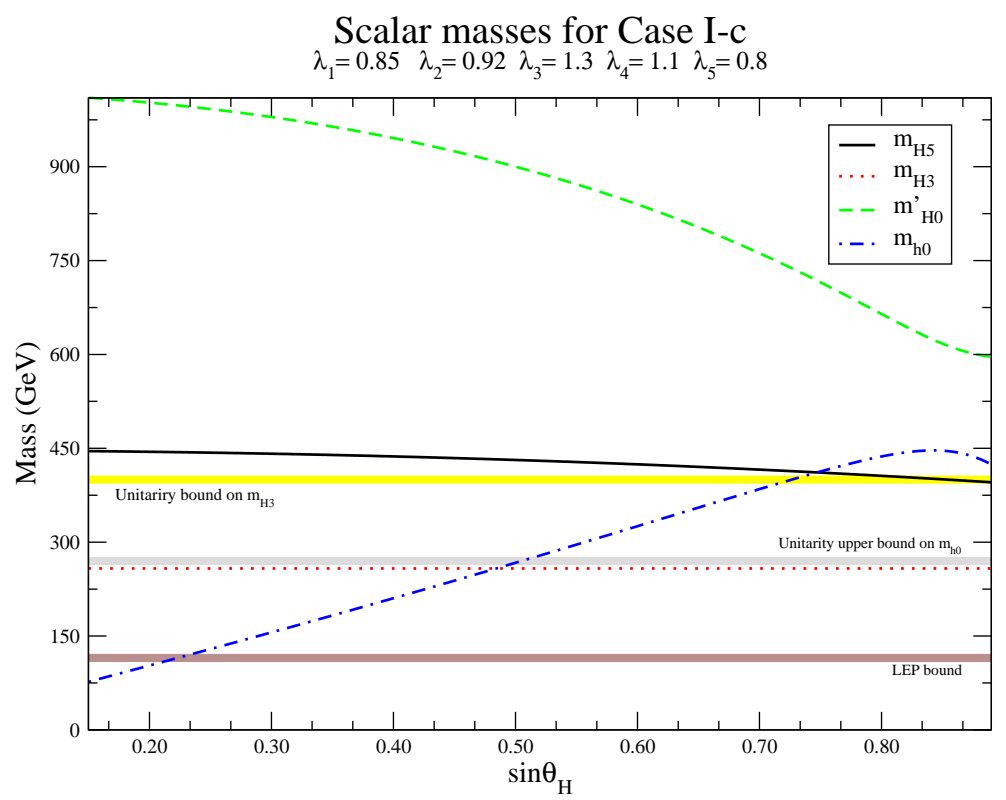

FIG. 3: Scalar mass spectrum for the case where there are no hierarchies among the parameters in the scalar potential. All parameters have intermediate values in the arbitrarily chosen $\mathrm{O}(1)$ range. 


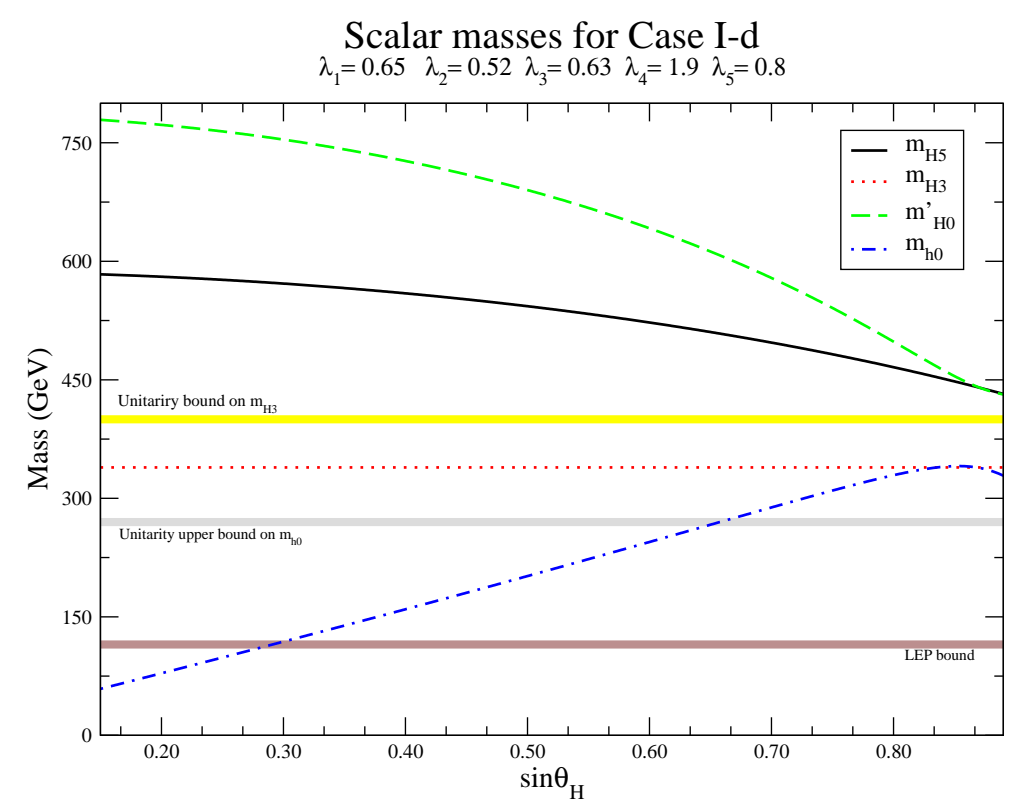

FIG. 4: Scalar mass spectrum for the case where there is a small hierarchy between $\lambda_{4}$ and the other parameters in the scalar potential. All parameters are in the $\mathrm{O}(1)$ range.

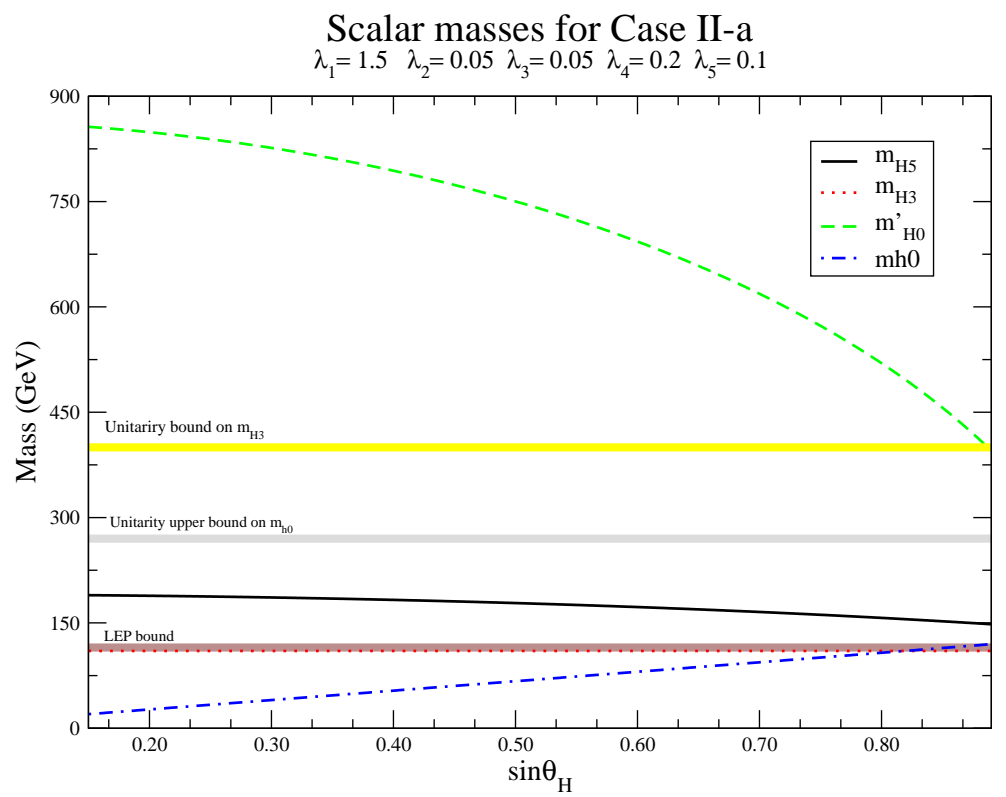

FIG. 5: Scalar mass spectrum for the case where there is a hierarchy between $\lambda_{1}$ and the other parameters in the scalar potential. $\lambda_{1}$ lies in the lower part of the $\mathrm{O}(1)$ range. This scenario satisfies all bounds for $\sin \theta_{H}>0.8$, except for LEP when $\sin \theta_{H}<0.8$ and can be used to analyze the possibility described in [11] of an undetected light scalar at LEP. 


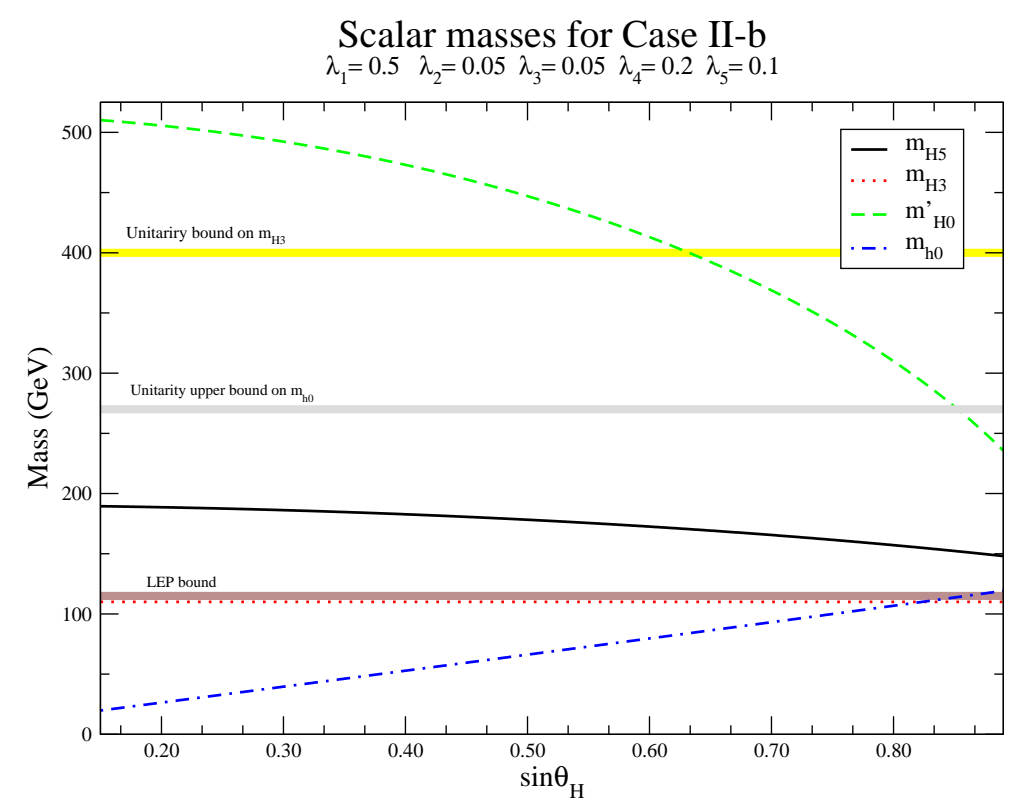

FIG. 6: Scalar mass spectrum for the case where there is a hierarchy between $\lambda_{1}$ and the other parameters in the scalar potential. $\lambda_{1}$ lies in the lower range of the $\mathrm{O}(1)$ range. This scenario satisfies all bounds except for LEP and can be used to analyze the possibility described in [11] of an undetected light scalar at LEP.
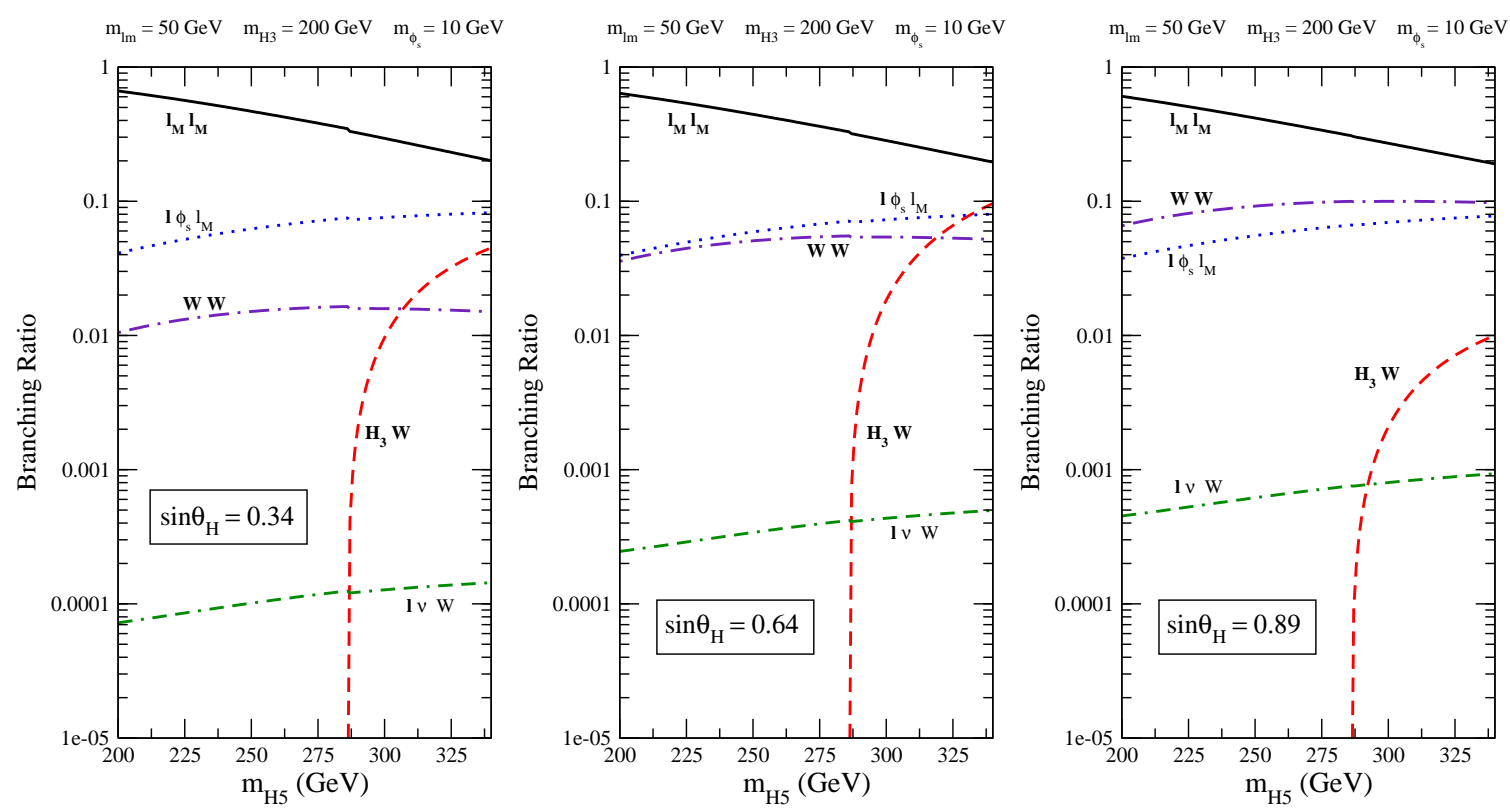

FIG. 7: Branching ratios for $\chi^{++}$as a function of its mass, for three different values of $\sin \theta_{H}$, and for a small $m_{l M}$. 

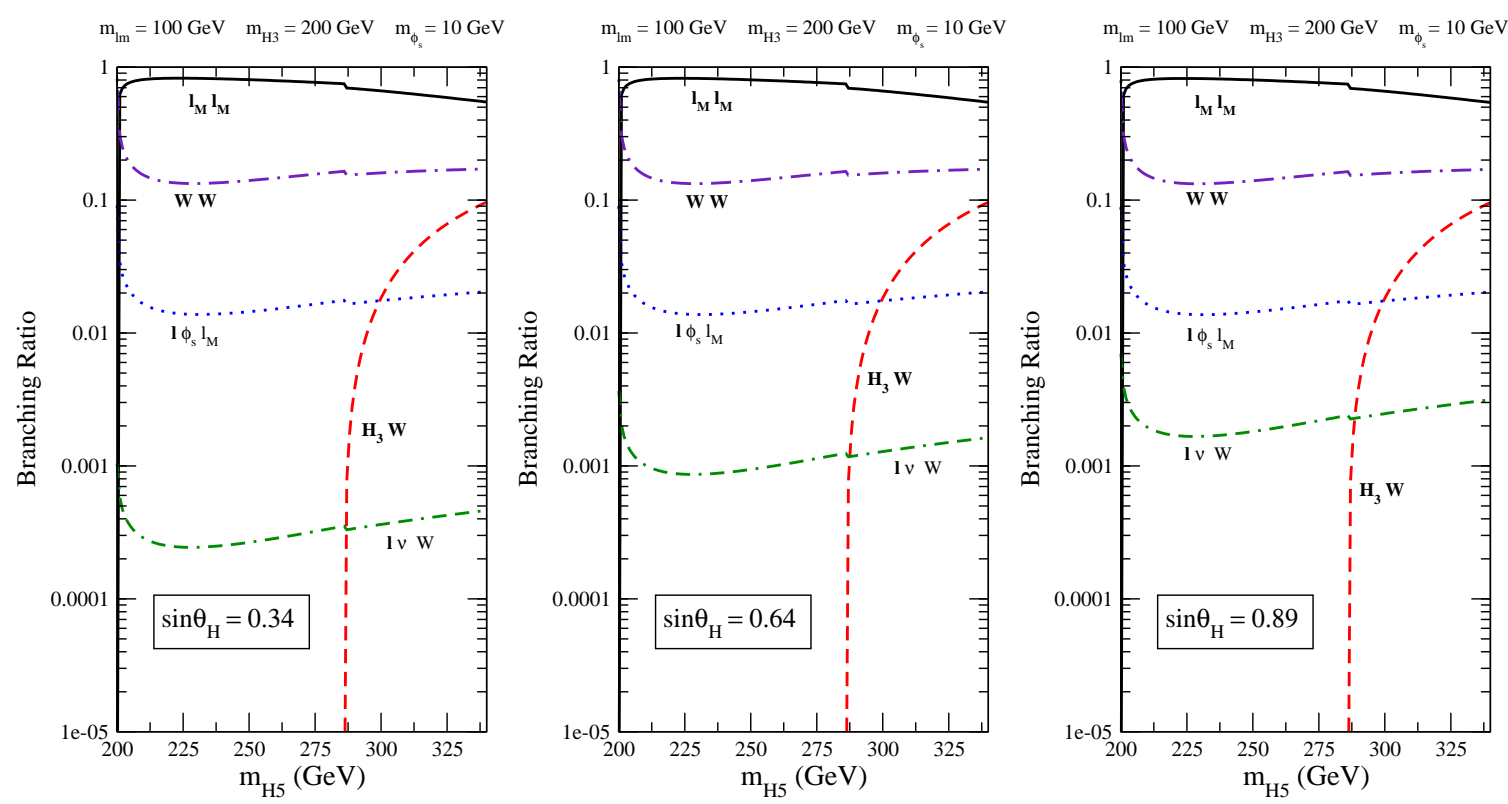

FIG. 8: Same as before but with a heavier $m_{l M}$. 